\title{
Differential phenotype of immune cells in blood and milk following pegylated granulocyte colony-stimulating factor therapy during a chronic Staphylococcus aureus infection in lactating Holsteins
}

\author{
E. J. Putz, ${ }^{1,2}$ J. M. Eder, ${ }^{1,3}$ T. A. Reinhardt, ${ }^{1}$ R. E. Sacco, ${ }^{1,3}$ E. Casas, ${ }^{1}$ and J. D. Lippolis ${ }^{1 *}$ \\ ${ }^{1}$ Ruminant Diseases and Immunology Research Unit, USDA Agricultural Research Service, National Animal Disease Center, Ames, IA 50010 \\ ${ }^{2}$ Oak Ridge Institute for Science and Education, Oak Ridge Associated Universities, Oak Ridge, TN 37830 \\ ${ }^{3}$ Immunobiology Interdepartmental Graduate Program, lowa State University, Ames 50011
}

\section{ABSTRACT}

Neutrophils are principal host innate immune cell responders to mastitis infections. Thus, therapies have been developed that target neutrophil expansion. This includes the neutrophil-stimulating cytokine granulocyte colony-stimulating factor (gCSF). Pegylated gCSF (PEG-gCSF; Imrestor, Elanco Animal Health, Greenfield, IN) has been shown to reduce the natural incidence of mastitis in periparturient cows in commercial settings and reduce severity of disease against experimental mastitis challenge. Pegylated gCSF stimulates neutrophil expansion but also induces changes in monocyte and lymphocyte circulating numbers, surface protein expression changes, or both. We hypothesized that PEG-gCSF modulates surface expression of monocytes and neutrophils and facilitates their migration to the mammary gland. We challenged 8 mid-lactation Holsteins with approximately $150 \mathrm{cfu}$ of Staphylococcus aureus (Newbould 305) in a single quarter via intramammary infusion. All animals developed chronic infections as assessed by bacteria counts and somatic cell counts (SCC). Ten to 16 wk postchallenge, 4 of the animals were treated with 2 subcutaneous injections of PEG-gCSF $7 \mathrm{~d}$ apart. Complete blood counts, SCC, bacterial counts, milk yield, feed intake, neutrophils extracellular trap analysis, and flow cytometric analyses of milk and blood samples were performed at indicated time points for $14 \mathrm{~d}$ after the first PEG-gCSF injection. The PEG-gCSF-treated cows had significantly increased numbers of blood neutrophils and lymphocytes compared with control cows. Flow cytometric analyses revealed increased surface expression of myeloperoxidase (MPO) on neutrophils and macrophages in milk but not in blood of treated cows. Neutrophils isolated

Received February 7, 2019.

Accepted June 10, 2019

*Corresponding author: john.lippolis@usda.gov from blood of PEG-gCSF-treated cows had decreased surface expression of CD62L (L-selectin) in blood, consistent with cell activation. Surprisingly, CD62L cell surface expression was increased on neutrophils and macrophages sourced from milk from treated animals compared with cells isolated from controls. The PEGgCSF-treated cows did not clear the $S$. aureus infection, nor did they significantly differ in SCC from controls. These findings provide evidence that PEG-gCSF therapy modifies cell surface expression of neutrophils and monocytes. However, although surface $\mathrm{MPO}^{+}$ cells accumulate in the mammary gland, the lack of bacterial control from these milk-derived cells suggests an incomplete role for PEG-gCSF treatment against chronic $S$. aureus infection and possibly chronic mammary infections in general.

Key words: chronic, mastitis, granulocyte colonystimulating factor, myeloperoxidase, L-selectin

\section{INTRODUCTION}

The economic costs and animal welfare concerns due to mastitis in dairy cattle continue to drive mastitis research into both prevention and nonantibiotic treatments. In the United States, mastitis results in a loss of approximately $\$ 2$ billion/yr to the dairy industry (USDA-APHIS, 2007). Mastitis symptoms can include reduced milk production, udder swelling and inflammation, poor milk quality, teat hardness, and secretory cell damage, thus can result in animal culling (Barkema et al., 2006; Yeiser et al., 2012). Although a wide breadth of pathogens can result in a mastitis infection, one of the most common is Staphylococcus aureus, which can present in acute or chronic forms (Taponen and Pyorala, 2009; Ryman et al., 2015; Ismail, 2017). Naturally occurring $S$. aureus infections have notably low cure rates (Linder et al., 2013), and disease severity and cure rate can be influenced by parity, quarter specificity, strain of $S$. aureus, and host genetic and environmental factors (Mallard et al., 1998; Barkema et al., 2006; Burvenich et 
al., 2007). One immune escape mechanism for S. aureus includes bacterial infiltration into the epithelial tissue of the mammary gland (Hensen et al., 2000a). Adherence and epithelial invasion, although strain specific, may contribute to the low antibiotic treatability and chronic capacities of $S$. aureus infections (Hensen et al., 2000b; Barkema et al., 2006). Different strains of $S$. aureus can produce varied inflammatory responses from the host as evidenced by differential cytokine production and proteomic expression from milk and serum (Kim et al., 2011). In this study, we used an experimental chronic $S$. aureus infection (Newbould 305) to study immune cell surface protein expression profiles and explore changes in these expression profiles between the periphery and milk and mammary gland.

Neutrophils are the dominant immune responders to mastitis infections. Neutrophils respond to inflammation signals and can infiltrate sites of infection (Fernandes et al., 1985; Kehrli et al., 1991a; Long et al., 2012). Activated neutrophils, known to migrate in response to bacterial threat to the mammary gland (Paape et al., 2003), are key producers of tumor necrosis factor- $\alpha$ (TNF- $\alpha)$, which plays an important role in inflammatory signaling and cross-communication with additional effector cells such as macrophages (Kobayashi et al., 2017). Once bacteria are encountered, neutrophils have numerous weapons at their disposal, including the ability to discharge their genomic DNA to produce neutrophil extracellular traps (NET), which have antimicrobial capabilities and physically ensnare various bacteria (Paape et al., 2003; Brinkmann et al., 2004; Lippolis et al., 2006). Neutrophils can also phagocytose bacteria, a process that results in respiratory burst production of oxidizing antimicrobials, which collectively can influence the severity of a mastitis infection through bacterial killing (Heyneman et al., 1990).

Although vaccines remain largely ineffective at controlling mastitis, the most common treatments are antibiotics (Barkema et al., 2006; Ismail, 2017). However, concerns about development of antimicrobial resistance from agricultural use of antibiotics are driving the effort to develop antibiotic alternatives. One alternative to antibiotics is immune modulation. The cytokine granulocyte colony-stimulating factor (gCSF) causes a transient neutrophilia (Canning et al., 2017; McDougall et al., 2017). Naturally, gCSF can be produced by activated monocytes and macrophages. Granulocyte colony-stimulating factor facilitates granulocyte cell maturation and activation, but it is also associated with the proliferation of myeloid progenitor cells from the bone marrow (Sieff, 1987; Kehrli et al., 1991a; Xu et al., 2000; Canning et al., 2017). In human and veterinary clinical practice, gCSF has been used to combat neutropenia or increase white blood cells (Kimura et al., 2014; Silvescu and Sackstein, 2014; Zinicola et al., 2018). Recently, a commercially available pegylated recombinant bovine gCSF (PEG-gCSF) treatment has been successful in lowering the incidence of naturally occurring mastitis of periparturient cattle (Canning et al., 2017; McDougall et al., 2017; Ruiz et al., 2017) and reducing the disease severity of an experimental Escherichia coli-induced mastitis challenge in mid-lactation animals (Powell et al., 2018).

A principal component of host defense against mastitis is the ability of systemic immune responders such as neutrophils to respond to inflammatory signals and, once activated, target the mammary gland (Paape et al., 2003). Although commonly recognized for its role in the production of reactive oxygen species, a glycosylated form of myeloperoxidase (MPO) has recently been established as a ligand for E-selectin (Silvescu and Sackstein, 2014). Myeloperoxidase can be found on the surface of monocytes and neutrophils and is upregulated following an activation stimulus, including gCSF (Kindzelskii et al., 2006; Powell et al., 2018). In dairy cattle, immune suppression during the critical periparturient period is associated with decreased expression of MPO (Cai et al., 1994; Lippolis et al., 2006) as well as reduced neutrophil function (Kehrli et al., 1991b; Mallard et al., 1998; Revelo et al., 2011).

Activation and maturation of neutrophils can be influenced by cytokines, chemokines, and similar inflammation signals, whereas the migration of cells to sites of infection is facilitated by adhesion molecules such as selectins (Long et al., 2012). L-Selectin (CD62L) is an adhesion molecule found on most leukocytes (Ivetic, 2018). Important L-selectin ligands include GlyCAM-1, ezrin-radixin-moesin proteins, and CD34 (Klinger et al., 2009; Ivetic, 2018). Additionally, in human neutrophils, L-selectin has been established to bind to E-selectin; however, this interaction is evidently species specific (Zöllner et al., 1997). Immune cells largely utilize surface L-selectin for rolling adhesion and surveillance. Upon interaction with an activating stimulus, L-selectin is rapidly cleaved from the cell surface by metalloproteases, primarily ADAM17 (Hansen et al., 2002; Smalley and Ley, 2005; Long et al., 2012; Ivetic, 2018). The ADAM17 enzyme has been implicated in a wide variety of cellular functions but was originally identified as the TNF- $\alpha$ converting enzyme. In addition to cleaving L-selectin, ADAM17 is responsible for cleaving TNF- $\alpha$ (Long et al., 2012; Ivetic, 2018). Work with cell lines and blood-derived cells has established that after an activation stimulus or disease challenge, there is a decrease in the cell surface expression of CD62L and, jointly, an increase in detectable shed soluble Lselectin (sL-selectin; Diez-Fraille et al., 2004; Wang et al., 2010; Ivetic, 2018). 
On cells isolated from milk, the behavior of surface Lselectin is less clear. In periparturient cattle, increased surface CD62L expression of lymphocytes was found in milk compared with the same cell type in circulation (Harp et al., 2004); additionally, a human comparison of sL-selectin found significantly higher levels of sLselectin in serum of lactating women than in the milk (Xyni et al., 2000). Experimental E. coli mastitis resulted in both blood and milk L-selectin expression loss, but the percentage of $\mathrm{CD}_{6} 2 \mathrm{~L}^{+}$polymorphonuclear neutrophils increased significantly in the milk (Diez-Fraille et al., 2004). In an S. aureus disease challenge study, there was a higher percentage of $\mathrm{CD} 62 \mathrm{~L}^{+}$leukocytes in the blood than the milk; however, the percentage of $\mathrm{CD}_{62 \mathrm{~L}^{+}}$polymorphonuclear neutrophils and $\mathrm{CD} 62 \mathrm{~L}^{+}$ macrophages was significantly higher in milk-sourced cells from S. aureus-infected cows than in those from healthy control cows (Nagahata et al., 2011).

Previously, our group established that circulating monocytes and neutrophils were activated by PEGgCSF therapy before mastitis challenge as indicated by increased surface expression of MPO, which suggests that activated cells were primed to respond to the inflammatory signals of the mammary gland, resulting in collective reduced severity of $E$. coli infection (Powell et al., 2018). This study used a chronic $S$. aureus model to explore the cell surface phenotypes between immune cells sourced from peripheral blood and mammary gland environments. We hypothesized that PEG-gCSF modulates surface expression of monocytes and neutrophils and facilitates their migration to the mammary gland. Although we provide evidence that $\mathrm{MPO}^{+}$cells accumulate in milk after PEG-gCSF treatment, we identified reduced bacterial killing and did not find additional evidence of antibacterial effector cell activity in milk-derived cells.

\section{MATERIALS AND METHODS}

\section{Experimental Design}

Eight Holstein cows were used in this study. Cows were in various lactations numbers ranging from first to third. Cows were divided into treatment groups to balance age and number of lactations. The National Animal Disease Center Animal Care and Use Committee approved all protocols. Cows were challenged in a single quarter with approximately $150 \mathrm{cfu}$ of $S$. aureus (experimental Newbold strain) via intramammary infusion in $2 \mathrm{~mL}$ of PBS. All cows developed chronic infections that were confirmed by SCC and bacterial count. Ten to 16 wk into chronic infection, 4 cows were injected in the neck with 2 subcutaneous doses of $2.7 \mathrm{~mL}$ of 15-mg PEG-gCSF (Imrestor, Elanco
Animal Health, Greenfield, IN) 7 d apart. Four cows were left untreated and served as chronically infected controls. However, a single control cow was diagnosed with naturally occurring, typed non- $S$. aureus mastitis in a non-S. aureus-infected quarter. This cow was treated with antibiotics and removed from the study. The 7 animals that completed the study ranged between 187 and 231 DIM. Milk yield, daily feed intake, SCC, bacterial counts, DNA in milk indicative of NET, complete blood counts (CBC), and flow cytometry of whole blood and isolated milk cells were collected as experimental parameters.

\section{SCC}

Milk samples were collected from experimental quarters on $\mathrm{d} 0,1,2,4,7,8,9,11$, and 14 following the first PEG-gCSF injection on d 0 (second PEG-gCSF injection after sample collection on d 7). The SCC samples were sent to and determined by Dairy Lab Services (Dubuque, IA).

\section{Milk Yield and Feed Intake}

All cows were milked twice daily using individual jar milking systems. Before milking, cows were washed with warm water, dried, and started milking or had samples collected by hand before milker placement. After milking, each teat was hand dipped in iodine before the cow was released from the parlor. Volume of milk produced at each milking was recorded. Individual feed intake data were collected for all cows using Calan feeding bunks (Broadbent Feeding System, American Calan, Northwood, NH), and cows were fed the farm's lactation diet (supportive of 36-46 kg of daily milk production). Each cow's daily feed ration was calculated as $10 \%$ over the previous week's average daily intake per animal. Remaining uneaten feed was weighed and recorded daily.

\section{Bacterial Count}

All animals were checked and were free of bacteria in all quarters before initial $S$. aureus challenge. Once chronic infection was established, cows were checked in all quarters $7 \mathrm{~d}$ prior and on $\mathrm{d} 0$ of this study. Colonyforming unit presence, morphology, and SCC were used to confirm that cows had only a single chronic experimental infection and only in the experimentally challenged quarter. For bacterial counts, milk was aseptically collected, serially diluted, and plated on trypticase soy agar with 5\% sheep blood plates (cat. no. 221261; BD Biosciences, San Jose, CA). Number 
of colonies was counted after overnight incubation at $37^{\circ} \mathrm{C}$.

\section{CBC and Hematology Slides}

Complete blood counts and blood smears were analyzed from EDTA whole blood collected from jugular venipuncture. The same blood samples were used for automated CBC (VetScan Hm5, Abaxis, Union City, $\mathrm{CA})$ and were mounted and stained for manual CBC. For blood smear slide analysis, 100 cells were characterized based on morphology and the proportion of major immune cell types (lymphocytes, monocytes, neutrophils, band neutrophils, eosinophils, and basophils) was recorded.

\section{Milk Cell Isolation and Milk Smears}

From each experimental quarter, $100 \mathrm{~mL}$ of milk was collected into $50-\mathrm{mL}$ tubes. Tubes were centrifuged at $4^{\circ} \mathrm{C}$ at $2,000 \times g$ for $40 \mathrm{~min}$. Milk cream and fat layers were scraped, and supernatant skim milk was discarded. Cell pellets were placed on ice and immediately resuspended in $2 \mathrm{~mL}$ of complete RPMI medium (supplemented with L-glutamine and 10\% fetal bovine serum). Cell solutions were counted (TC20 automated cell counter, BioRad, Hercules, CA) in duplicate and total yields of live cells were calculated. Aliquots were then stained (see below) for flow cytometry analysis and were mounted on slides for microscopic analysis and a manual cell count (see above). For slide analysis, 100 cells were characterized based on morphology and the proportion of major immune cell types (lymphocytes, monocytes, neutrophils, band neutrophils, eosinophils, and basophils) recorded.

\section{Neutrophil Extracellular Trap Analysis}

From each experimental quarter, $100 \mathrm{~mL}$ of milk was centrifuged for $45 \mathrm{~min}$ at $4^{\circ} \mathrm{C}$ at $10,000 \times \mathrm{g}$. The milk cream and fat layers were washed once with PBS and protease inhibitor and frozen. At the end of the study, milk fats were thawed by time point and stained to quantify neutrophil extracellular trap (NET) mean fluorescent intensity (MFI). Weighed milk fats were stained with $5 \mu M$ Sytox Orange (cat. no S11368; Invitrogen, Eugene, OR). After 10 min of incubation, samples were washed with NET buffer (10 $\mathrm{m} M$ Tris, $150 \mathrm{~m} M \mathrm{KCl}, 250 \mathrm{~m} M$ sucrose, $2 \mathrm{mM} \mathrm{MgCl}_{2}$, $\mathrm{pH} 7.5$ ) and centrifuged at $1,500 \times g$ at $4^{\circ} \mathrm{C}$ for $10 \mathrm{~min}$. Milk fats were then scraped and resuspended in NET buffer. For each animal, control milk fats were treated with DNase (Benzonase, cat. no. E8263-25KU; Sigma-Aldrich, St. Louis, MO) and then stained as described above. The NET MFI was calculated for each animal as raw MFI minus DNase-treated MFI. All samples were analyzed in duplicate on a plate reader (FlexStation3; Molecular Devices, Sunnyvale, CA) at $570 \mathrm{~nm}$.

\section{Flow Cytometry}

Cell surface staining was completed on both EDTAtreated whole blood and isolated milk cells (see isolation protocol above). Panel design and antibodies used are shown in Table 1. Briefly, independent primary, secondary, and directly conjugated antibody cocktails were added and incubated for $15 \mathrm{~min}$ at room temperature in the dark. In between staining steps, samples were washed with flow buffer (cat. no. 420201; BioLegend, San Diego, CA), were centrifuged, had supernatant removed, and were resuspend in residual buffer. A Becton Dickinson (Franklin Lakes, NJ) LSR II flow cytometer was used for all samples, and data were evaluated using FlowJo software (FlowJo LLC, Ashland, OR).

Milk Cells. Milk cell samples utilized a livedead stain (1:100 diluted Zombie Yellow, cat. no. 423103/423104; BioLegend) before panel staining. Each milk sample was washed in PBS and had $10^{6}$ milk cells (see above) incubated with live-dead stain for $20 \mathrm{~min}$

Table 1. Primary and secondary antibodies used to characterize whole blood and isolated milk cells

\begin{tabular}{lll}
\hline Marker & Primary antibodies & Secondary antibodies \\
\hline Myeloperoxidase & Cat. no. VPA00193; BioRad, Hercules, CA & $\begin{array}{l}\text { Rat anti-rabbit Ig-PE, cat. no. 4065-09; } \\
\text { SouthernBiotech, Birmingham, AL }\end{array}$ \\
CD62L (L-selectin) & Cat. no. 304824; BioLegend, San Diego, CA & Directly conjugated \\
CD45 & Cat. no. BOV2039; Monoclonal Antibody Center, & Brilliant violet 421 anti-mouse IgG2a, cat. no. 407117; \\
& Washington State University, Pullman & BioLegend \\
CD138 & Cat. no. BOV2068; Monoclonal Antibody Center, & APC/Cy7 anti-mouse IgG1 antibody, cat. no. 406620; \\
& Washington State University & BioLegend \\
CD14 & Cat. no. 301812; BioLegend & Directly conjugated \\
Pan-lymphocyte & Cat. no. BOV2071; Monoclonal Antibody Center, & BUV395 rat anti-mouse IgG3, cat. no. 744138; BD \\
& Washington State University & Biosciences, San Jose, CA \\
\hline
\end{tabular}


with rocking at room temperature in the dark. Samples were washed with buffer and then stained as described.

Whole Blood. Blood flow data were collected using $50 \mu \mathrm{L}$ of whole blood from EDTA collection tubes. After staining, $25 \mu \mathrm{L}$ of counting beads (cat. no. ACBP100-10; Spherotech AccuCount Particles, Lake Forest, IL) was added to blood cell samples, and suspensions were subjected to a 30-min lysis (cat. no. 349202; FACS Lysing Solution, San Jose, CA).

Gating Strategy. See Supplemental Figures S1 (blood) and S2 (milk; https://doi.org/10.3168/jds.2019 -16448) for visual gating strategy. Milk and blood cells were gated to evaluate only live single cells. All cells (both milk and blood) were evaluated for single cell or doublet discrimination and were additionally gated for CD45. Additionally, autofluorescence in the FITC 488 channel was used to identify and remove eosinophils as a contaminant of the neutrophil population (Dorward et al., 2013). From CD $45^{+}$cells, determination of monocytes and macrophages was based on forward and side scatter and expression of CD14. From CD $45^{+}$ cells, neutrophils were CD $138^{+}$and gated with forward and side scatter for granulocytes. Surface expression of MPO and CD62L was individually assessed within the $\mathrm{CD} 14^{+}$monocyte and macrophage and neutrophil gates.

\section{TNF- $\alpha$ and sL-Selectin ELISA}

Serum samples were obtained by jugular venipuncture with 8-mL serum Vacutainer tubes (cat. no. 367988; BD Biosciences, San Jose, CA). Tubes were incubated for $30 \mathrm{~min}$ at $37^{\circ} \mathrm{C}$ and centrifuged for $20 \mathrm{~min}$ at 1,500 $\times g$. Serum was pipetted into aliquots and frozen at $-80^{\circ} \mathrm{C}$ until it was thawed for use. Skim milk samples were prepared from $100 \mathrm{~mL}$ of milk from experimental quarters. Whole milk was centrifuged for $45 \mathrm{~min}$ at $4^{\circ} \mathrm{C}$ at $10,000 \times g$, after which the top cream and fat layer was removed and the bottom cell pellet was avoided. Skim milk samples were also aliquoted and stored at $-80^{\circ} \mathrm{C}$ until thawed for use. All samples were analyzed undiluted. The sL-selectin concentrations were determined using a bovine-specific competitive ELISA kit (cat. no. ABIN993381; Antibodies Online, Atlanta, GA) used to the manufacturer's specifications. Samples were read in duplicate at an absorbance of $450 \mathrm{~nm}$ via a plate reader (FlexStation3, Molecular Devices). Serum and skim milk samples were also evaluated for TNF- $\alpha$ concentration via bovine-specific TNF- $\alpha$ ELISA kit (cat. no. VS0285B-002; KingFisher Biotech, Saint Paul, $\mathrm{MN}$ ) according to the manufacturer's specifications and analyzed in duplicate on a plate reader at $450 \mathrm{~nm}$.

\section{Statistical Analysis}

$\mathrm{R}$ [packages base (stats), car, effect, lsmeans, ggplots2, and lme4; R Core Team, 2014] was used to fit multiple linear regression models including fixed effects of experimental day, PEG-gCSF treatment, and source of cells (blood or milk) if relevant. No random effects were fit. Some models described below also evaluated the ANOVA interaction of treatment and day for significance. For certain treatment and day contrasts, a pairwise comparison of least squares means (LSM) was used. $P$-values $\leq 0.05$ were determined to be significant. All error bars represented standard errors.

Daily Milk Yield and Feed Intake. For both milk yield $(\mathrm{kg} / \mathrm{d})$ and feed intake (percentage of feed offered that was consumed), fixed effects of treatment and day as well treatment $x$ day interaction were evaluated for significance.

SCC Analysis and Bacterial Load Analysis. Bacterial colony-forming units were counted by hand. Counts were then base 10 log-transformed. Fixed effects of PEG-gCSF treatment and experimental day along with treatment $x$ day interaction were evaluated for significance. Specific day LSM contrasts for PEGgCSF-treated and control cows were also analyzed.

NET Analysis. For mean NET MFI, fixed effects of PEG-gCSF treatment and experimental day along with treatment $\times$ day interaction were evaluated for significance. Specific day LSM contrasts for PEG-gCSFtreated and control cows were analyzed. Collectively timed responses (injection day, $24 \mathrm{~h}$ postinjection, and $48 \mathrm{~h}$ postinjection) were also evaluated as fixed effects.

$C B C$ Analysis (Blood and Milk). For the automated CBC analysis, cell numbers were individually analyzed for circulating neutrophils, monocytes, and lymphocytes. Manual counts were also done on blood smear slides to determine the proportion of mature versus immature band neutrophils. The percentage band and percentage mature neutrophils were applied to automated CBC total neutrophil counts to determine band and mature counts. Manual counts of milk cells were only analyzed as percentages of each cell type. Fixed effects of PEG-gCSF treatment and experimental day along with treatment $x$ day interaction were evaluated for significance. Specific day LSM contrasts for PEG-gCSF-treated and control cows were also analyzed.

Flow Cytometry MFI Analysis. Geometric mean static values were determined by FlowJo software within $\mathrm{CD} 14^{+}$and neutrophil gates (see above) for MPO and CD62L, respectively. Fixed effects of PEG-gCSF treatment and experimental day along with treatment $\times$ 
day interaction were evaluated for significance. Specific day LSM contrasts for PEG-gCSF-treated and control cows were also analyzed.

sL-Selectin and TNF- $\alpha$ ELISA Analysis. For both sL-selectin and TNF- $\alpha$, fixed effects of PEG-gCSF treatment and experimental day along with treatment $x$ day interaction were evaluated for significance. Soluble L-selectin concentrations were evaluated as the change (in $\mathrm{ng} / \mathrm{mL}$ ) from d 0. Specific day LSM contrasts for PEG-gCSF-treated and control cows were analyzed.

\section{RESULTS}

All cows were confirmed to have a single-quarter chronic $S$. aureus infection by bacterial counts. The PEG-gCSF treatment of cows with an $S$. aureus chronic infection did not result in clearance of the infection. The PEG-gCSF-treated cows did not differ from control animals in percentage of offered feed consumed (92.9 \pm $1.2 \%$ vs. $94.1 \pm 1.3 \%$, respectively). Similarly, daily milk yields did not differ significantly between treated $(29.6 \pm 1.0 \mathrm{~kg} / \mathrm{d})$ and control $(30.7 \pm 1.1 \mathrm{~kg} / \mathrm{d})$ cows.

Our data show significantly higher bacterial counts with PEG-gCSF treatment at 1 time point (d 7); however, the biological significance of that increase is questionable (Figure 1A). What was clear was that PEGgCSF treatment did not result in control of the infection or result in bacterial clearance. There were no effects of PEG-gCSF on SCC and no significant interactions of treatment and experimental day at any time points. Overall, across experimental days, $\log _{10}$-transformed SCC numbers were not significantly different between PEG-gCSF-treated cows $\left(6.3 \pm 0.1 \log _{10}\right.$ SCC $)$ and untreated cows $\left(6.3 \pm 0.1 \log _{10} \mathrm{SCC} ; P=0.98\right.$; Figure 1B). As another informative marker of milk neutrophil behavior, we quantified the presence of DNA, which is indicative of NET in the milk fat from treated and nontreated cattle (Figure 1C). The PEG-gCSF-treated cows had a reduced NET MFI, collectively, $24 \mathrm{~h}$ postinjection $(P=0.03)$. However, this effect was significant only when the time point after injection was evaluated as a fixed effect, meaning that combined effects from d 1 and 8 were significantly decreased compared with chronically infected controls. This significant difference disappeared by $48 \mathrm{~h}$ postinjection (d 2 and $9 ; P=$ 0.80). Specifically, NET MFI significantly decreased from d 7 to $\mathrm{d} 8$ in milk fat from treated cows $(P=0.03)$ following the second PEG-gCSF injection (Figure 1C).

Treatment with PEG-gCSF had significant effects on blood neutrophil and lymphocyte counts $(P<0.01)$ as evidenced by CBC values (Figure 2). The PEG-gCSFtreated animals maintained significantly higher blood neutrophil numbers at all time points following PEGgCSF injection $(P<0.01 ;$ Figure $2 \mathrm{~A})$. Lymphocyte numbers were significantly increased only in treated cows following the second PEG-gCSF injection on d 8 , d $9(P<0.01)$, and d $14(P=0.01$; Figure $2 \mathrm{~B})$. Circulating monocyte numbers did not differ significantly between treatment groups (Figure 2C). Similar to previous findings (Powell et al., 2018), blood slide hematology analysis showed that band neutrophils appeared in significant numbers $4 \mathrm{~d}$ after the initial PEGgCSF treatment (Figure 2D), whereas mature neutrophils respond by $24 \mathrm{~h}$ posttreatment (Figure 2E). Band neutrophil numbers were significantly higher $4 \mathrm{~d}$ after the first injection (d $4 ; P<0.01)$ and 1 and $2 \mathrm{~d}$ after the second injection (d 8 and $9 ; P<0.01$; Figure $2 \mathrm{D}$ ), and circulating mature neutrophils were significantly increased d 1 through $14(P<0.01$; Figure 2E).

Manual counts of centrifuged milk cells yielded percentages of lymphocytes, monocytes, and neutrophils present (Figure 3). No significant effects of PEG-gCSF were found, nor were any significant contrasts between treatment groups by experimental day. Band neutrophils were present only in milk from PEG-gCSF-treated animals. Band neutrophils were found in a single treated animal on d 4 and 7 and in all treated cows on d $8(1.25 \pm 1.1 \%)$.

To compare immune cell subsets between circulating blood and milk environments, we used flow cytometry to evaluate the cell surface expression of MPO on $\mathrm{CD} 14^{+}$monocytes (blood) or macrophages (milk; Figure 4) and neutrophils (Figure 5) by analyzing the LSM of the MFI of the marker of interest as well as by representative histogram. Contrary to the prior $E$. coli study, we saw no significant increase in surface MPO expression on circulating monocytes in the blood of PEG-gCSF-treated and control cows at any time point (Figure 4A, C). However, milk macrophages from PEG-gCSF-treated animals had significantly higher surface MPO expression on d $2(P=0.02)$ and $\mathrm{d} 4(P$ $<0.01$ ) compared with nontreated controls (Figure 4B, D). Similarly, no significant differences of surface MPO expression were found between blood neutrophils from treated and untreated cows (Figure 5A, C), but neutrophils from milk of PEG-gCSF-treated animals had significantly increased MPO expression on d $4(P=$ $0.04)$, d $8(P<0.01)$, and d $9(P<0.01$; Figure 5B, D).

To further provide information about the expression profile of cells between blood and milk, we also used the same flow cytometry approach to examine the changes in surface expression of CD62L (L-selectin) on monocytes and macrophages (Figure 6) and neutrophils (Figure 7). Consistent with MPO expression, circulating monocytes had no differences in CD62L surface expression between treatment groups (Figure $6 \mathrm{~A}, \mathrm{C})$; however, macrophages from milk of PEG-gCSF cows had significantly increased CD62L expression on 

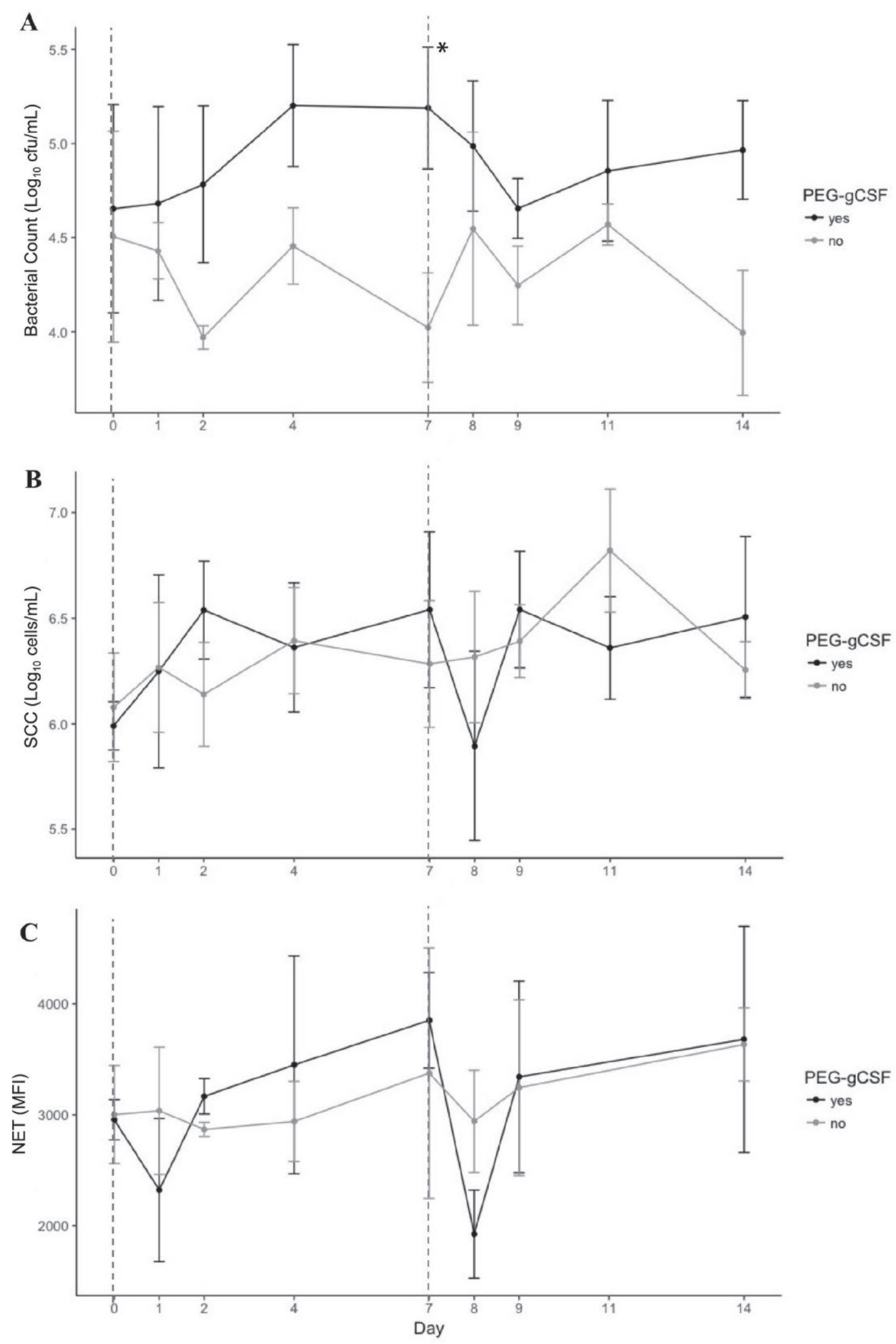

Figure 1. Milk parameters of pegylated granulocyte colony-stimulating factor (PEG-gCSF)-treated cows (black lines) had a significant increase in bacterial load, no differences in SCC, and decreases in neutrophil extracellular traps (NET) compared with nontreated, chronically infected control cows (gray lines). (A) $\log _{10}$-transformed bacterial counts; (B) $\log _{10}$-transformed SCC; (C) DNA in milk fat indicative of NET, shown as the LSM of NET mean fluorescent intensity (MFI). Vertical dashed lines show days of PEG-gCSF injection for treated animals. Error bars denote SE; asterisk denotes significant differences between PEG-gCSF-treated and untreated groups with $P$-value $\leq 0.05$. 

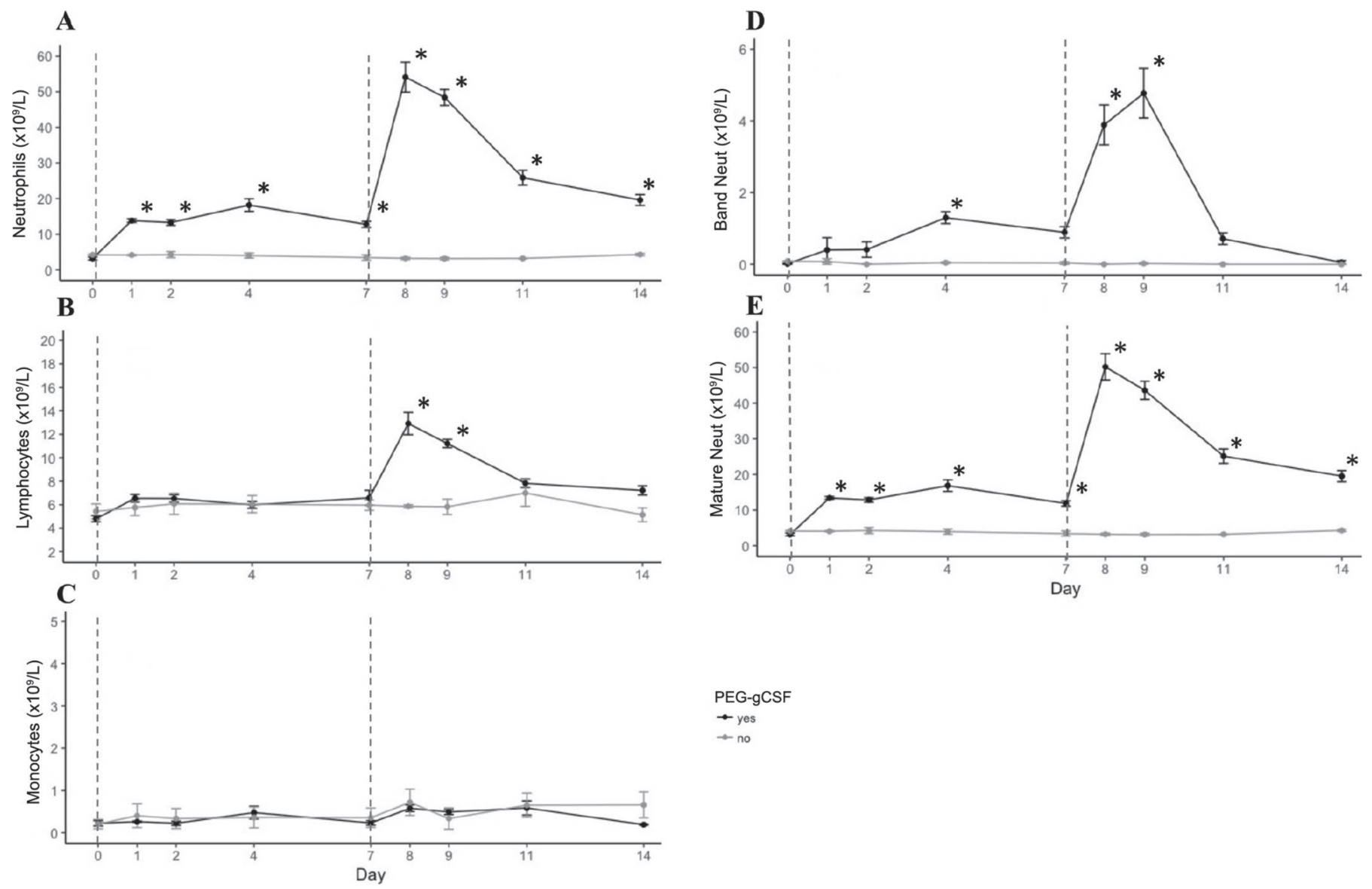

Figure 2. Complete blood counts (CBC) show increases in neutrophils and lymphocytes for pegylated granulocyte colony-stimulating factor (PEG-gCSF)-treated cows (black lines) compared with controls (gray lines). Automated CBC are shown for (A) neutrophils, (B) lymphocytes, and $(\mathrm{C})$ monocytes. Manual CBC were performed on blood slides to determine proportions of (D) band neutrophils (Neut) compared with (E) mature neutrophils. Vertical dashed lines show days of PEG-gCSF injection for treated animals. Error bars denote SE; asterisks denote significant differences between PEG-gCSF-treated and untreated groups with $P$-value $\leq 0.05$.

d $4(P<0.01)$ and d $8(P<0.01$; Figure $6 \mathrm{~B}, \mathrm{D})$. Circulating neutrophils from the blood of PEG-gCSFtreated cows exhibited significantly decreased CD62L expression on $\mathrm{d} 4(P=0.02)$, d $8(P<0.01)$, and $\mathrm{d}$ $9(P<0.01)$ compared with neutrophils from control cows (Figure 7A, C). In contrast, neutrophils derived from PEG-gCSF-treated cow milk had significantly increased CD62L expression on d $4(P=0.02)$ and $\mathrm{d}$ $8(P<0.01)$ compared with those from control cattle (Figure 7B, D).

The changes in surface expression of L-selectin between peripheral blood- and milk-derived cells prompted us to examine shed sL-selectin protein levels in the serum and skim milk (Figure 8). In serum, normalized sL-selectin concentrations (differences from d 0) were significantly increased in PEG-gCSF serum samples on d $4(P=0.02)$ and d $9(P<0.01)$ compared with control cow samples (Figure 8A). In skim milk samples, significantly less sL-selectin was found compared with serum samples $(63.64 \pm 2.56$ vs. $675.92 \pm 57.16 \mathrm{ng} / \mathrm{mL}$, respectively; $P<0.01$ ), and there were no significant differences between treated and untreated skim milk samples overall $(P=0.73)$ or by day $(P=0.96$; Figure $8 \mathrm{~B})$.

In addition to L-selectin, we examined another notable ADAM17 cleaving target, TNF- $\alpha$, which we quantified in serum and skim milk (Figure 9). Similar to sL-selectin results, we found significantly less TNF- $\alpha$ in the skim milk than in serum $(P<0.01$; Figure 9A, B). In the serum and the skim milk, there was no significant effect of PEG-gCSF, nor any significant experimental day contrasts between treated and control groups.

\section{DISCUSSION}

Our hypothesis was that PEG-gCSF modulates the surface expression of monocytes and neutrophils and facilitates their migration to the mammary gland. We 
established that treatment with PEG-gCSF changes the cell surface protein expression profile of immune cell subsets in the blood and the milk. These cell surface proteins with expression changes are known or hypothesized to be involved in the targeting of immune cells to a site of infection, which is consistent with our hypothesis. Subcutaneous injection of PEGgCSF resulted in circulating neutrophil expansion and shedding of L-selectin from blood-derived neutrophils, which confirms that PEG-gCSF therapy does elicit neutrophil responses. Furthermore, the accumulation of increased surface expression of MPO on milk-sourced macrophages and neutrophils suggests that PEG-gC-
SF-specific responses can be identified directly in the mammary gland.

As was shown previously, PEG-gCSF therapy resulted in a significant increase in circulating neutrophils in peripheral blood following the second PEG-gCSF injection (Figure 2A; Powell et al., 2018; Van Schyndel et al., 2018). However, a smaller increase in neutrophil numbers was observed in the chronic $S$. aureus model in response to the first PEG-gCSF injection, which contrasts with the observations in our previous $E$. coli study, where animals were treated before disease challenge (Powell et al., 2018). Manual counts of blood smears revealed that PEG-gCSF-treated cows have

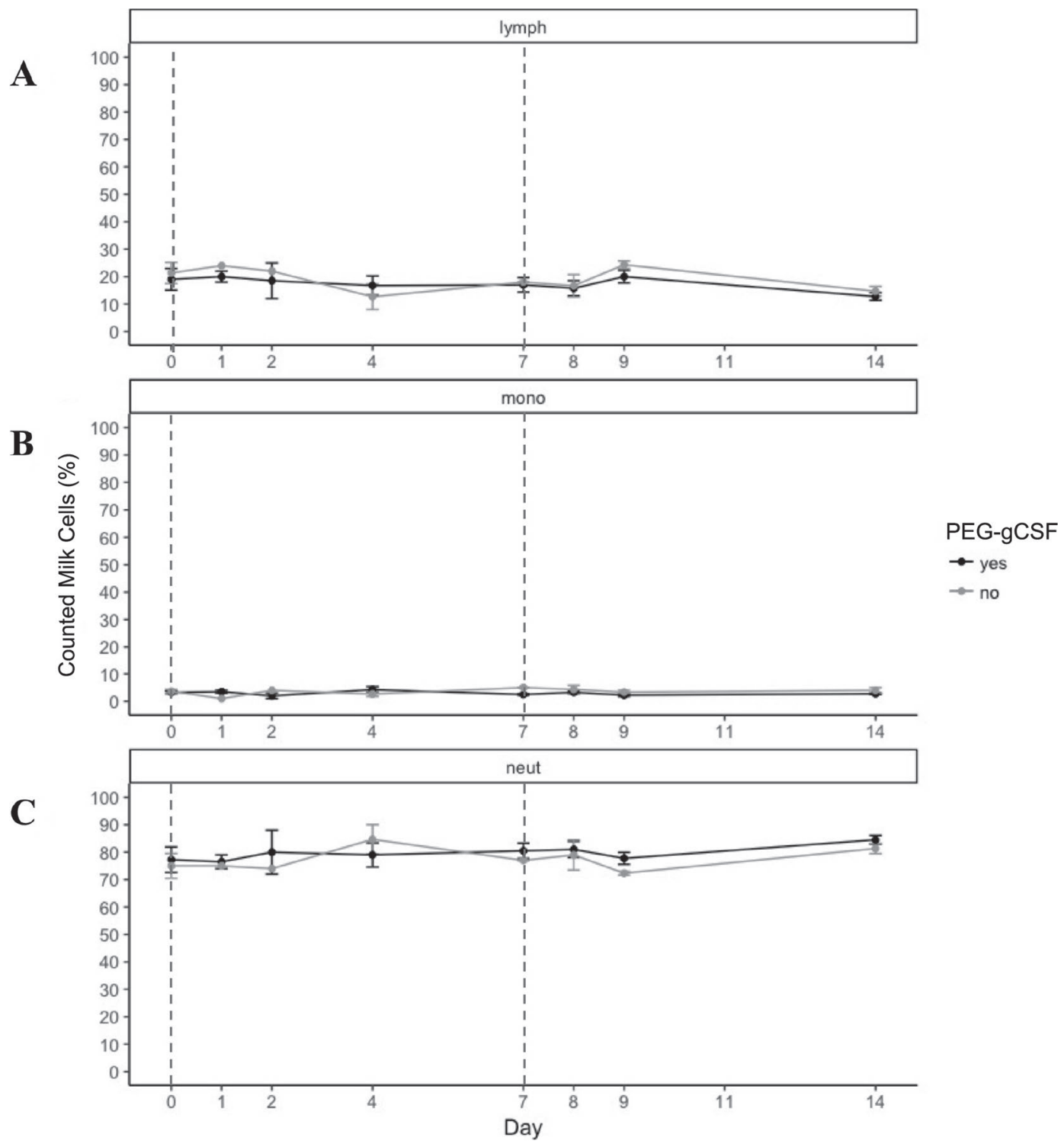

Figure 3. Manual milk counts of pegylated granulocyte colony-stimulating factor (PEG-gCSF) cows (black lines) and control cows (gray lines). Manual counts of milk smears were made from $100 \mathrm{~mL}$ of whole milk that was centrifuged to condense cells (see Materials and Methods). Of 100 total counted cells, the percentage of (A) lymphocytes (lymph), (B) monocytes (mono), and (C) total neutrophils (neut) are depicted. Vertical dashed lines show days of PEG-gCSF injection for treated animals. Error bars denote SE. 

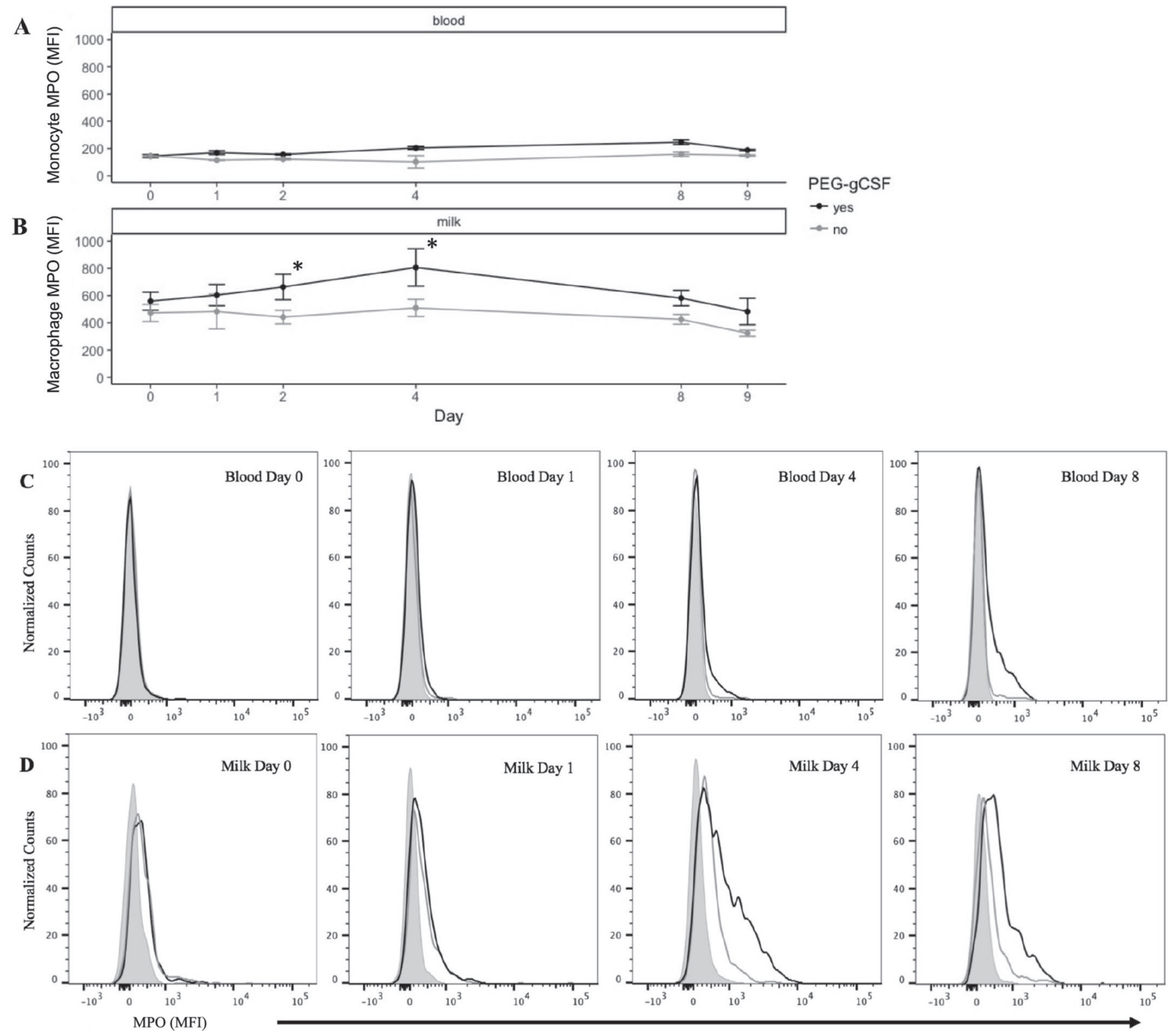

Figure 4. Monocyte and macrophage surface expression of myeloperoxidase (MPO). Mean fluorescent intensity (MFI) was quantified for MPO expression on the surface of $\mathrm{CD} 14^{+}$monocytes from (A) blood and macrophages from (B) milk. Representative histograms are also depicted from (C) blood and (D) milk. The black lines indicate cells from pegylated granulocyte colony-stimulating factor (PEG-gCSF)-treated cows, and gray lines indicate cells from control animals. Solid gray histogram represents fluorescence minus one control for marker of interest. Error bars denote SE; asterisks denote significant differences between PEG-gCSF-treated and untreated groups with $P$-value $\leq 0.05$.

an appearance of band (immature) neutrophils in the blood, peaking after the second PEG-gCSF injection (Figure 2D), which is consistent with periparturient work showing an increase in circulating band neutrophils (Van Schyndel et al., 2018). Smears of milk cells identified a small yet significant increase in band neutrophils after the second injection on d 8, suggesting that the vast majority of neutrophils found in milk are mature. Unlike in the 2018 E. coli study (Powell et al.,
2018), we did not see an increase in peripheral monocyte numbers following the first injection of PEG-gCSF (Figure 2C). However, highly surface expressing $\mathrm{MPO}^{+}$ macrophages appeared earlier in the mammary gland (d 2) than neutrophils (d 4) in treated animals (Figures 4 and 5, respectively).

To better characterize cells of interest, we used flow cytometry to examine the expression of surface markers of interest on neutrophils and monocytes or 
macrophages isolated from blood and milk. Previously, our group established that when healthy animals were treated with PEG-gCSF, blood neutrophils and monocytes exhibited increased surface MPO expression (Powell et al., 2018). When those healthy animals were then challenged with intramammary E. coli, these $\mathrm{MPO}^{+}$populations were no longer detectable in the blood, suggesting that the surface $\mathrm{MPO}^{+}$neutrophils and monocytes were responding to inflammatory signals and migrating to the mammary gland. It is known that surface MPO can act as a ligand for E-selectin (Silvescu and Sackstein, 2014) and that blocking E- selectin under activating conditions reduces neutrophil adherence (Maddox et al., 1999). The findings presented here are consistent with this concept of MPO acting as a ligand for E-selectin. Chronically infected cows treated with PEG-gCSF had no significantly detectable increases in surface MPO on blood monocytes (Figure 4) or neutrophils (Figure 5). However, in the milk, both macrophages (Figure 4) and neutrophils (Figure 5) had increased surface MPO expression on cells isolated from the milk of PEG-gCSF-treated cows. These findings in combination with the results of the previous study suggest that PEG-gCSF alters MPO

A
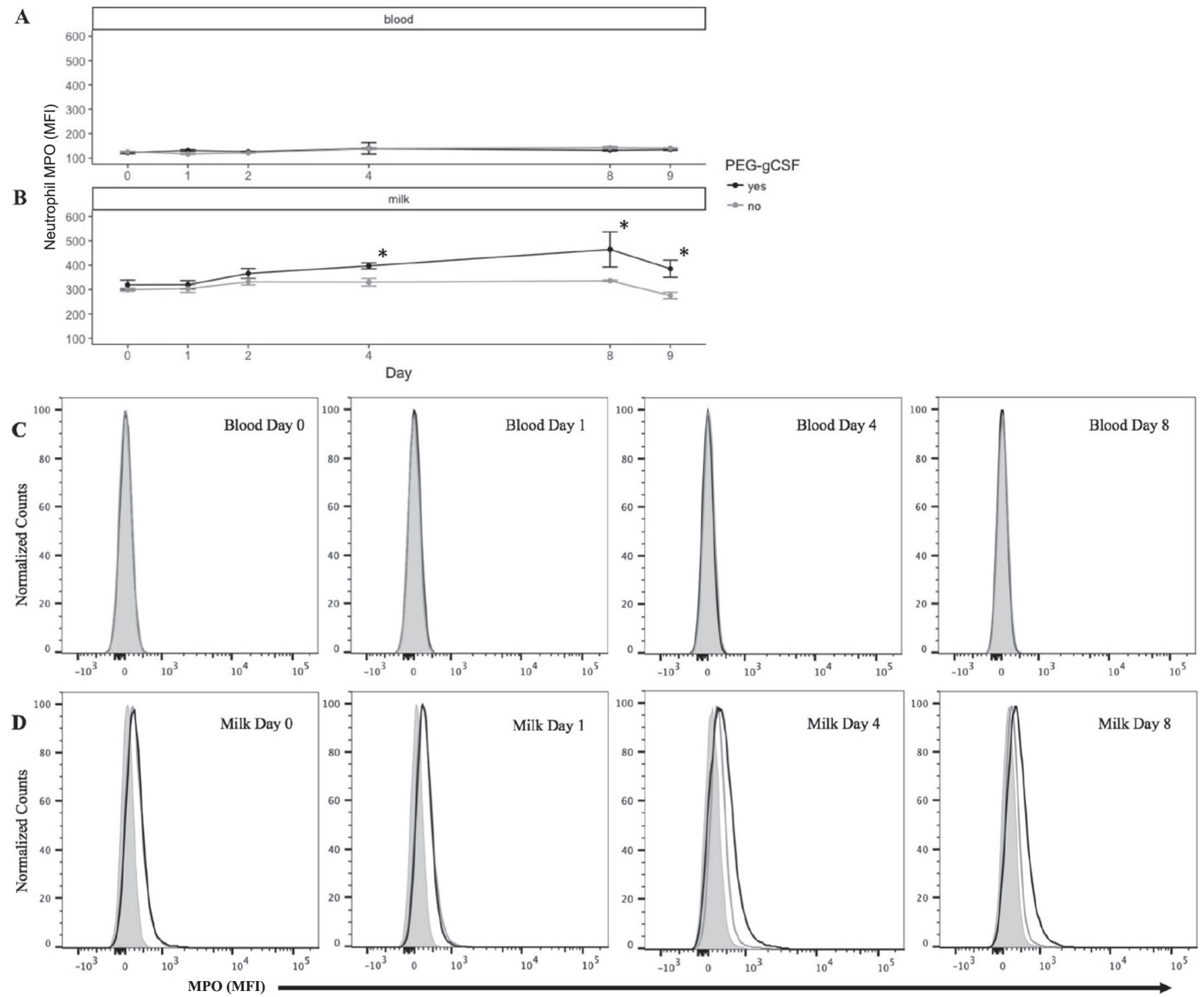

Figure 5. Neutrophil surface expression of myeloperoxidase (MPO). Mean fluorescent intensity (MFI) was quantified for MPO expression on the surface of CD138 ${ }^{+}$neutrophils from (A) blood and (B) milk. Representative histograms are also depicted from (C) blood and (D) milk. The black lines indicate cells from pegylated granulocyte colony-stimulating factor (PEG-gCSF)-treated cows, and gray lines indicate cells from control animals. Solid gray histogram represents fluorescence minus one control for marker of interest. Error bars denote SE; asterisks denote significant differences between PEG-gCSF-treated and untreated groups with $P$-value $\leq 0.05$. 

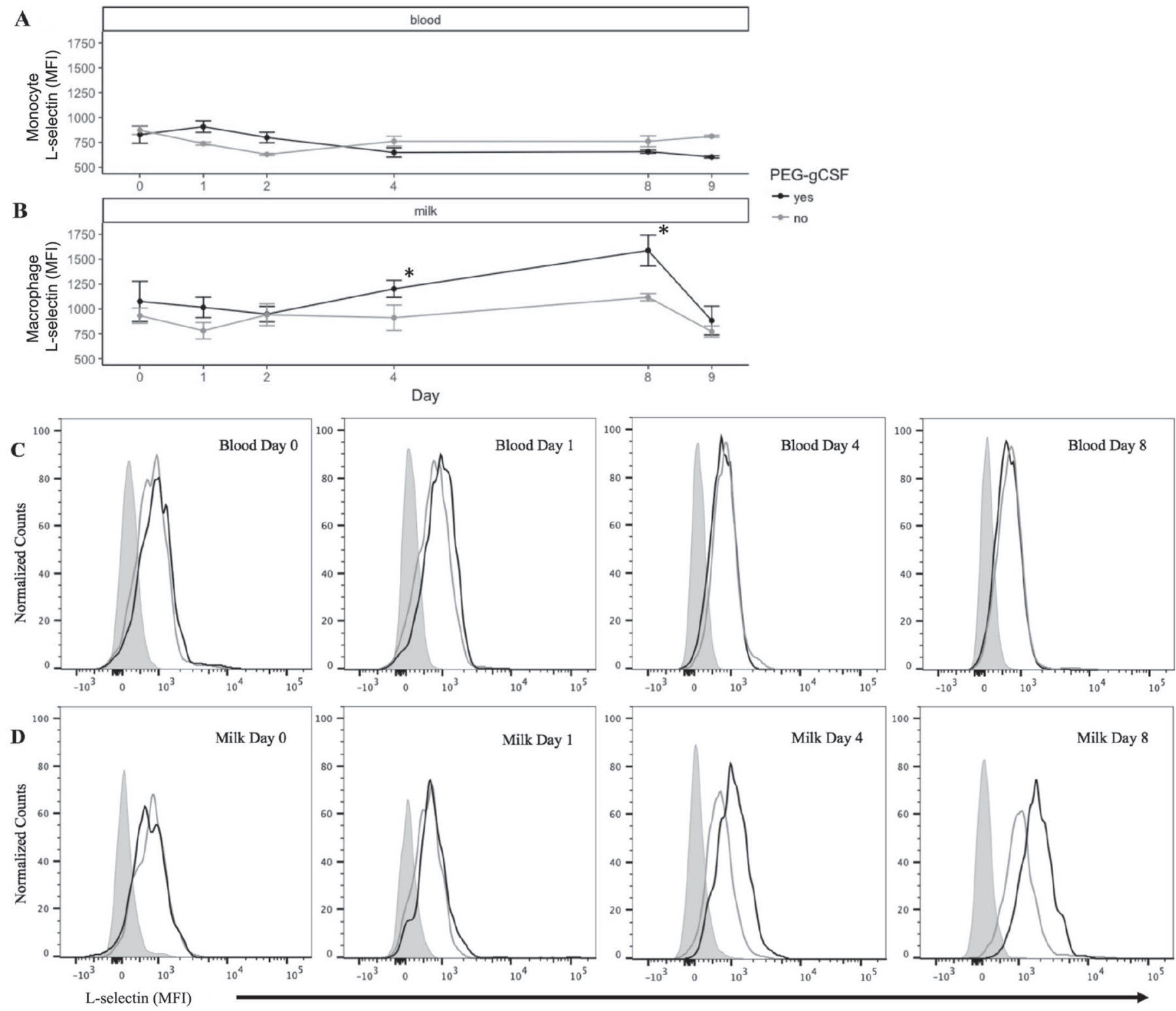

Figure 6. Monocyte and macrophage surface expression of CD62L (L-selectin). Mean fluorescent intensity (MFI) was quantified for CD62L expression on the surface of $\mathrm{CD}_{14}^{+}$monocytes from (A) blood and macrophages from (B) milk. Representative histograms are also depicted from (C) blood and (D) milk. The black lines indicate cells from pegylated granulocyte colony-stimulating factor (PEG-gCSF)-treated cows, and gray lines indicate cells from control animals. Solid gray histogram represents fluorescence minus one control for marker of interest. Error bars denote SE; asterisks denote significant differences between PEG-gCSF-treated and untreated groups with $P$-value $\leq 0.05$.

surface expression of neutrophils and monocytes and is associated with an accumulation of $\mathrm{MPO}^{+}$cells in a mammary gland undergoing a mastitis infection.

We sought to further describe cell behavior by assessing other informative markers in addition to MPO. A decrease in surface expression of L-selectin is recognized as an indicator of myeloid cell activation (Keeney et al., 1993; Hansen et al., 2002; Ivetic, 2018). It is well established that neutrophils shed surface L-selectin in response to numerous stimuli, including meningococ- cal bacteria (Heyderman et al., 1999), fMLP protein (Kuhns et al., 1995), damage-associated molecular patterns (Hazeldine et al., 2015), and other inflammatory stimuli (Killock and Ivetic, 2010; Ivetic, 2018). Consistent with the literature, we saw decreased surface expression of L-selectin on neutrophils in the blood of PEGgCSF-treated cows posttreatment, suggesting cleavage of surface L-selectin (Figure 7). In further support of increased L-selectin cleavage, indicative of neutrophil activation in the blood, increased concentrations of sL- 

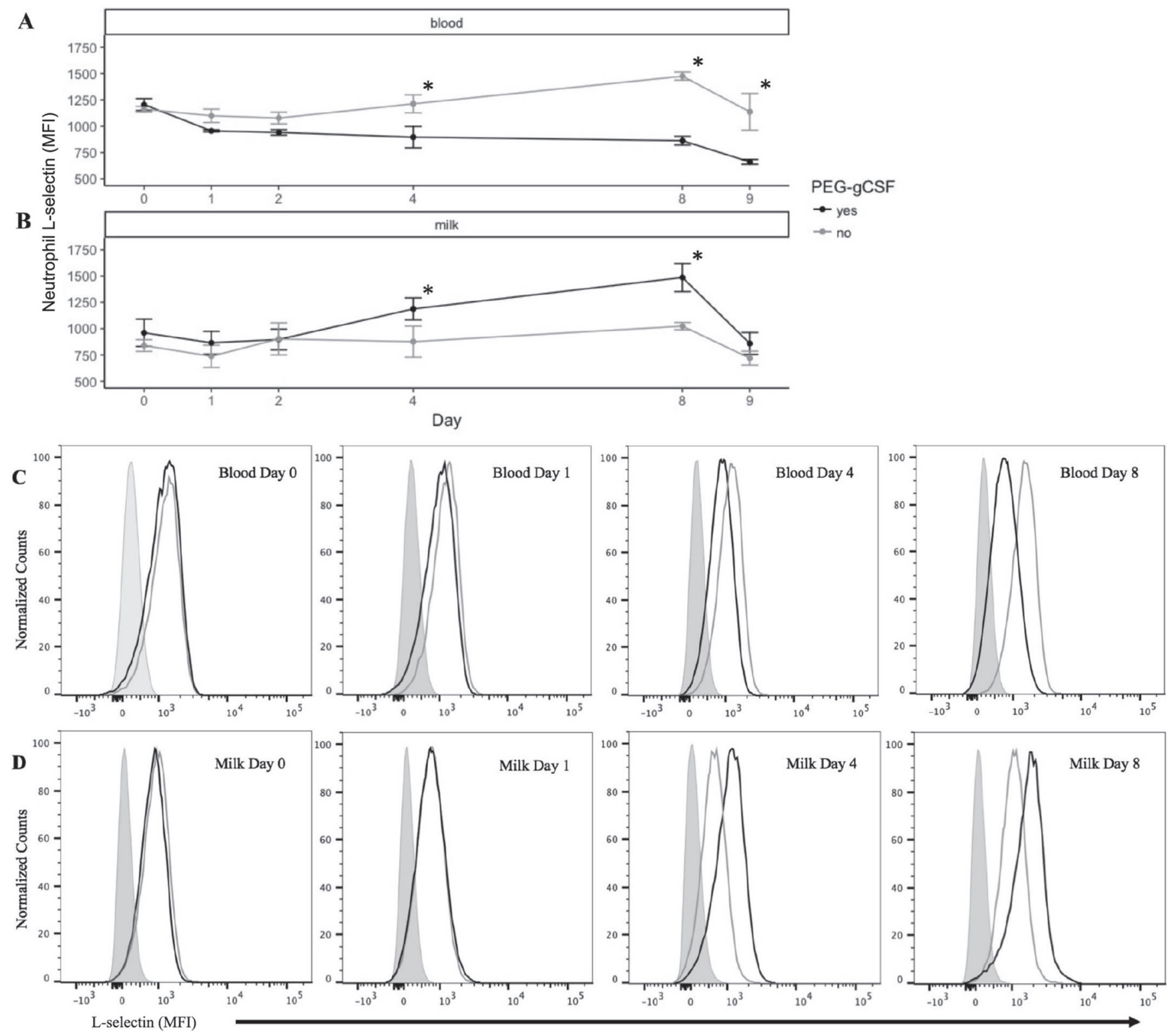

Figure 7. Neutrophil surface expression of CD62L (L-selectin). Mean fluorescent intensity (MFI) was quantified for CD62L expression on the surface of CD138 $8^{+}$neutrophils from (A) blood and (B) milk. Representative histograms are also depicted from (C) blood and (D) milk. The black lines indicate cells from pegylated granulocyte colony-stimulating factor (PEG-gCSF)-treated cows, and gray lines indicate cells from control animals. Solid gray histogram represents fluorescence minus one control for marker of interest. Error bars denote SE; asterisks denote significant differences between PEG-gCSF-treated and untreated groups with $P$-value $\leq 0.05$.

selectin were detected in the serum of PEG-gCSF-treated cows compared with serum from untreated animals (Figure 8A). There were no changes in blood monocyte surface expression of CD62L between treatment groups despite the fact that monocytes are known to shed Lselectin in response to activation (Hansen et al., 2002). Contrary to peripheral blood neutrophils, milk-derived macrophages and neutrophils from PEG-gCSF-treated cows expressed increased surface L-selectin (Figures 6 and 7, respectively). This finding was also supported by the lower concentrations of sL-selectin in skim milk compared with serum levels and was largely unchanged between milk treatment groups over the experimental time points (Figure 8B). In the literature, there is evidence that L-selectin expression levels are increased on $\mathrm{T}$ cells found in nonlymphoid tissue compared with lymphoid organs (Klinger et al., 2009), which could be relevant to the nonlymphoid mammary gland. Data 
A)

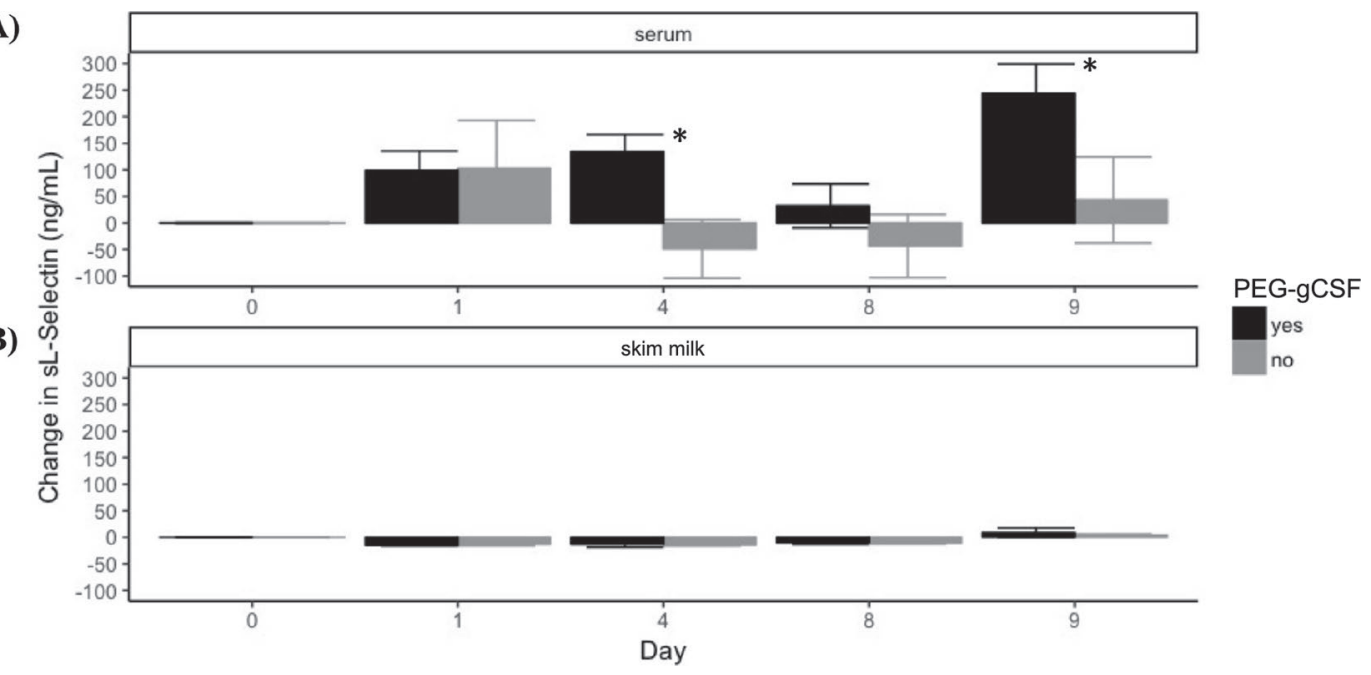

Figure 8. Changes in concentrations of soluble L-selectin (sL-selectin) in blood and milk environments. Cleaved sL-selectin (differences from d 0) was quantified in (A) serum and (B) skim milk. The black bars indicate samples from pegylated granulocyte colony-stimulating factor (PEG-gCSF)-treated cows, and gray bars indicate samples from control animals. Error bars denote SE; asterisks denote significant differences between PEG-gCSF-treated and untreated groups with $P$-value $\leq 0.05$.

on milk-derived lymphocytes have shown an increased percentage of CD62L positive cells found in the milk compared with the same cell type in the blood (Harp et al., 2004), whereas other literature has established that neutrophils and macrophages in milk decrease Lselectin expression in response to activation (Keeney et al., 1993).

Interestingly, both sL-selectin and $\mathrm{TNF}-\alpha$ are primarily products of the same protease, ADAM17 (Ivetic, 2018). This is of particular interest because TNF- $\alpha$ is an important acute phase cytokine produced by neutrophils. Similar to sL-selectin levels, TNF- $\alpha$ concentrations were virtually nondetectable in skim milk compared with serum levels in both PEG-gCSF-treated and control animals (Figure 9). Reduced concentrations of milk TNF- $\alpha$ compared with blood levels of TNF- $\alpha$ have been established, and TNF- $\alpha$ levels in milk have low (0.04) correlation with naturally occurring mastitis (Denholm et al., 2018). However, TNF- $\alpha$ levels have been shown to increase in milk following endotoxin challenge (Paape et al., 2002). This suggests a need for future work to examine the function and role of

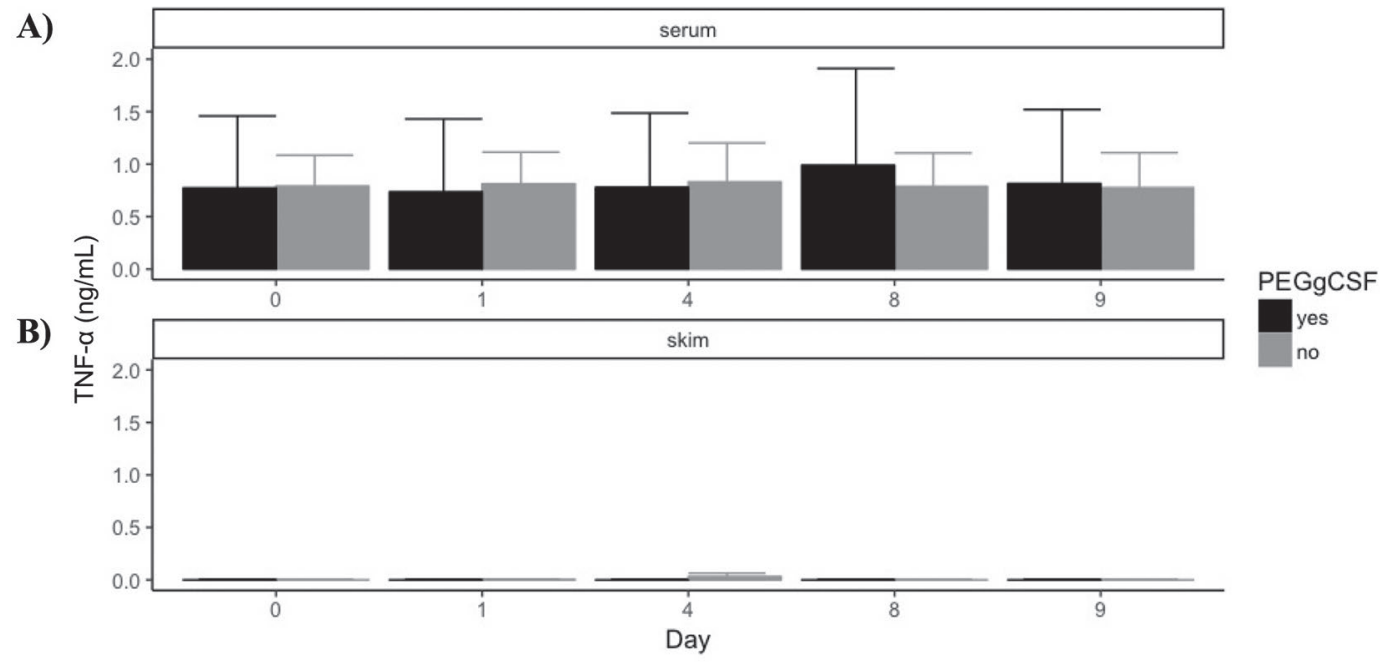

Figure 9. Concentrations of tumor necrosis factor- $\alpha$ (TNF- $\alpha$ ) in blood and milk environments. TNF- $\alpha$ was quantified in (A) serum and (B) skim milk. The black bars indicate samples from pegylated granulocyte colony-stimulating factor (PEG-gCSF)-treated cows, and gray bars indicate samples from control animals. Error bars denote SE; asterisks denote significant differences between PEG-gCSF-treated and untreated groups with $P$-value $\leq 0.05$. 
ADAM17 in the mammary gland environment during a chronic infection.

Despite the observation that cells with higher $\mathrm{MPO}^{+}$ surface expression accumulate in the milk, we did not find any difference in SCC values between the treated and control groups (Figure 1B). This is consistent with periparturient data (Canning et al., 2017) and our 2018 $E$. coli study that showed that SCC remain markedly similar between PEG-gCSF-treated and untreated cows despite a pronounced increase in circulating blood cells (Powell et al., 2018). Also supporting the idea that milk immune cell subsets are not profoundly altered by PEG-gCSF treatment are the milk smear counts that show no variation in percentage of neutrophils, macrophages, or lymphocytes between milk samples of treated and untreated animals (Figure 3). Part of the explanation for the occurrence of chronic mastitis infections could be that bacteria escape immune detection by entering mammary epithelial cells and, importantly, reducing inflammatory signaling (Günther et al., 2017). Our data suggest that some inflammatory signals occur because SCC are still elevated in infected compared with control quarters (data not shown). An important facet of future work will be characterizing which inflammatory signals are present and which are absent within any given mammary gland environment.

One striking observation from this study is that the pronounced neutrophilia in circulating blood is not seen in the milk as assessed by SCC (Figure 1B), NET content (Figure 1C), or change in immune cell percentages of milk-derived cells (Figure 3). It is plausible that an increase in SCC could be masked by highly activated neutrophils immediately releasing NET, resulting in the death of the neutrophil and thus not being counted in SCC values. However, our NET data showed no increase in NET in PEG-gCSF-treated animals. In our previous study, we observed a significant increase in NET from milk fat from PEG-gCSF-treated animals $24 \mathrm{~h}$ after an experimental $E$. coli disease challenge. In contrast, we found that during a chronic $S$. aureus infection there was no difference between treated and control animals except for a transient decrease in NET immediately following the PEG-gCSF injection (Figure 1C). The NET MFI reached approximately 3,500 MFI at the peak of an E. coli challenge (Powell et al., 2018), whereas the d 0 MFI for NET content consisted of 2,955.3 \pm 612.5 MFI for PEG-gCSF-treated cows and 3,001.832 \pm 707.3 MFI for control cows undergoing a chronic experimental $S$. aureus injection, providing further evidence of an existing and active infection.

Bacterial counts by day were not significantly different between treated and nontreated animals with the exception of $\mathrm{d} 7$. It can be noted that bacterial load has a high level of variability during $S$. aureus infections and that host and environmental factors play a complex synergistic role in disease severity and response (Shoshani et al., 2000; Barkema et al., 2006; Rivas et al., 2007). Given the level of variability seen in the bacterial count data and the number of animals in this experiment, we do not believe that this is a significant biological difference, and it was not the focus of these experiments. It has also been established that the SCC may not be correlated with bacterial counts in an S. aureus infection (Shoshani et al., 2000). In addition, previous studies also suggest that chronic or subclinical stage of $S$. aureus infection may affect the response to GM-CSF (Takahashi et al., 2004). Earlystage animals with experimental $S$. aureus challenge in the first month of infection had decreased SCC and significantly decreased bacterial counts after a single GM-CSF injection compared with late-stage animals that had infections for a minimum of 2 mo (Takahashi et al., 2004).

It is reasonable to presume that the mammary gland microenvironment may contribute to changes in cell surface phenotypes. Selectins are well characterized for the roles they play while immune cells traffic to and from lymph nodes. It is conceivable that the process of migration into the mammary gland involves different signaling or activation cascades, and the passage of primed immune cells from peripheral blood to the mammary gland modifies leukocyte surface markers. It has been established that the process of diapedesis alone has reduced the ability of neutrophils to phagocytose bacteria and produce reactive oxygen species (Smits et al., 1999). Additionally, it is known that neutrophils exhibit reduced bactericidal activity with the ingestion of milk fat and casein (Russell and Reiter, 1975; Paape and Guidry, 1977).

The data presented herein establish that surface MPO and L-selectin expression of macrophages and neutrophils are altered by PEG-gCSF treatment and facilitates, in part, accumulation of surface $\mathrm{MPO}^{+}$cells into the mammary gland. However, our results also found that PEG-gCSF treatment is associated with reduced pathogen defense, potentially due to the induction of an inadequate or inappropriate host response in the mammary gland environment. Overall, this suggests that although PEG-gCSF elicited neutrophil- and macrophage-specific responses, the increase in bacterial counts and inability to clear a chronic $S$. aureus infection demonstrates that PEG-gCSF is not a practical therapeutic solution for this modeled chronic infection.

\section{ACKNOWLEDGMENTS}

Special thanks go to Duane Zimmerman, Tera Nyholm, Adrienne Shircliff, Sam Humphrey, Judith 
Stasko, Nate Horman, and the dairy animal care staff at the National Animal Disease Center (USDA/ARS) in Ames, Iowa. Mention of trade names or commercial products in this article is solely for the purpose of providing specific information and does not imply recommendations or endorsement by the USDA. The USDA was the sole funder for this research. The USDA determines the research priorities of all research that it funds. Publication of research is subject to review of USDA officials.

\section{REFERENCES}

Barkema, H. W., Y. H. Schukken, and R. N. Zadoks. 2006. Invited review: The role of cow, pathogen, and treatment regimen in the therapeutic success of bovine Staphylococcus aureus mastitis. J. Dairy Sci. 89:1877-1895.

Brinkmann, V., U. Reichard, C. Goosmann, B. Fauler, Y. Uhlemann, D. S. Weiss, Y. Weinrauch, and A. Zychlinsky. 2004. Neutrophil extracellular traps kill bacteria. Science 303:1532-1535.

Burvenich, C., D. D. Bannerman, J. D. Lippolis, L. Peelman, B. J. Nonnecke, M. E. Kehrli Jr., and M. J. Paape. 2007. Cumulative physiological events influence the inflammatory response of the bovine udder to Escherichia coli infections during the transition period. J. Dairy Sci. 90(Suppl. 1):E39-E54.

Cai, T. Q., P. G. Weston, L. A. Lund, B. Brodie, D. J. McKenna, and W. C. Wagner. 1994. Association between neutrophil functions and periparturient disorders in cows. Am. J. Vet. Res. 55:934-943.

Canning, P., R. Hassfurther, T. TerHune, K. Rogers, S. Abbott, and D. Kolb. 2017. Efficacy and clinical safety of pegbovigrastim for preventing naturally occurring clinical mastitis in periparturient primiparous and multiparous cows on US commercial dairies. J. Dairy Sci. 100:6504-6515.

Denholm, S. J., T. N. McNeilly, G. Banos, M. P. Coffey, G. C. Russell, A. Bagnall, M. C. Mitchell, and E. Wall. 2018. Immune-associated traits measured in milk of Holstein-Friesian cows as proxies for blood serum measurements. J. Dairy Sci. 101:10248-10258.

Diez-Fraille, A., J. Mehrzad, E. Meyer, L. Duchateau, and C. Burvenich. 2004. Comparison of L-selectin and Mac-1 expression on blood and milk neutrophils during experimental Escherichia coli-induced mastitis in cows. Am. J. Vet. Res. 65:1164-1171.

Dorward, D. A., C. D. Lucas, A. L. Alessandri, J. A. Marwick, F. Rossi, I. Dransfield, C. Haslett, K. Dhaliwal, and A. G. Rossi. 2013. Technical advance: Autofluorescence-based sorting: Rapid and nonperturbing isolation of ultrapure neutrophils to determine cytokine production. J. Leukoc. Biol. 94:193-202.

Fernandes, A. C., R. Anderson, and G. J. Ras. 1985. An objective filter-based, enzymatic method for the in vivo measurement of the migration of human polymorphonuclear leucocytes. J. Immunol. Methods 83:259-271.

Günther, J., W. Petzl, I. Bauer, S. Ponsuksili, H. Zerbe, H. J. Schuberth, R. M. Brunner, and H. M. Seyfert. 2017. Differentiating Staphylococcus aureus from Escherichia coli mastitis: S. aureus triggers unbalanced immune-dampening and host cell invasion immediately after udder infection. Sci. Rep. 7:4811.

Hansen, P. S., S. B. Petersen, K. Varning, and H. Nielsen. 2002. Additive effects of Helicobacter pylori lipopolysaccharide and proteins in monocyte inflammatory responses. Scand. J. Gastroenterol. 37:765-771.

Harp, J. A., T. E. Waters, and J. P. Goff. 2004. Lymphocyte subsets and adhesion molecule expression in milk and blood of periparturient dairy cattle. Vet. Immunol. Immunopathol. 102:9-17.

Hazeldine, J., P. Hampson, F. A. Opoku, M. Foster, and J. M. Lord. 2015. $N$-Formyl peptides drive mitochondrial damage associated molecular pattern induced neutrophil activation through ERK1/2 and P38 MAP kinase signalling pathways. Injury 46:975-984.
Hensen, S. M., M. J. Pavicic, J. A. Lohuis, J. A. de Hoog, and B. Poutrel. 2000a. Location of Staphylococcus aureus within the experimentally infected bovine udder and the expression of capsular polysaccharide type 5 in situ. J. Dairy Sci. 83:1966-1975.

Hensen, S. M., M. J. Pavicic, J. A. Lohuis, and B. Poutrel. 2000b. Use of bovine primary mammary epithelial cells for the comparison of adherence and invasion ability of Staphylococcus aureus strains. J. Dairy Sci. 83:418-429.

Heyderman, R. S., C. A. Ison, M. Peakman, M. Levin, and N. J. Klein. 1999. Neutrophil response to Neisseria meningitidis: Inhibition of adhesion molecule expression and phagocytosis by recombinant bactericidal/permeability-increasing protein (rBPI21). J. Infect. Dis. 179:1288-1292.

Heyneman, R., C. Burvenich, and R. Vercauteren. 1990. Interaction between the respiratory burst activity of neutrophil leukocytes and experimentally induced Escherichia coli mastitis in cows. J. Dairy Sci. 73:985-994.

Ismail, Z. B. 2017. Mastitis vaccines in dairy cows: Recent developments and recommendations of application. Vet. World 10:10571062.

Ivetic, A. 2018. A head-to-tail view of L-selectin and its impact on neutrophil behaviour. Cell Tissue Res. 371:437-453.

Keeney, S. E., F. C. Schmalstieg, K. H. Palkowetz, H. E. Rudloff, B. M. Le, and A. S. Goldman. 1993. Activated neutrophils and neutrophil activators in human milk: Increased expression of CD11b and decreased expression of L-selectin. J. Leukoc. Biol. 54:97-104.

Kehrli, M. E., Jr., J. S. Cullor, and S. C. Nickerson. 1991a. Immunobiology of hematopoietic colony-stimulating factors: Potential application to disease prevention in the bovine. J. Dairy Sci. 74:4399-4412.

Kehrli, M. E., Jr., J. P. Goff, M. G. Stevens, and T. C. Boone. 1991b. Effects of granulocyte colony-stimulating factor administration to periparturient cows on neutrophils and bacterial shedding. J. Dairy Sci. 74:2448-2458.

Killock, D. J., and A. Ivetic. 2010. The cytoplasmic domains of TNFalpha-converting enzyme (TACE/ADAM17) and L-selectin are regulated differently by p38 MAPK and PKC to promote ectodomain shedding. Biochem. J. 428:293-304.

Kim, Y., H. Atalla, B. Mallard, C. Robert, and N. Karrow. 2011. Changes in Holstein cow milk and serum proteins during intramammary infection with three different strains of Staphylococcus aureus. BMC Vet. Res. 7:51.

Kimura, K., J. P. Goff, P. Canning, C. Wang, and J. A. Roth. 2014. Effect of recombinant bovine granulocyte colony-stimulating factor covalently bound to polyethylene glycol injection on neutrophil number and function in periparturient dairy cows. J. Dairy Sci. 97:4842-4851.

Kindzelskii, A. L., A. J. Clark, J. Espinoza, N. Maeda, Y. Aratani, R. Romero, and H. R. Petty. 2006. Myeloperoxidase accumulates at the neutrophil surface and enhances cell metabolism and oxidant release during pregnancy. Eur. J. Immunol. 36:1619-1628.

Klinger, A., A. Gebert, K. Bieber, K. Kalies, A. Ager, E. B. Bell, and J. Westermann. 2009. Cyclical expression of L-selectin (CD62L) by recirculating T cells. Int. Immunol. 21:443-455.

Kobayashi, S. D., N. Malachowa, and F. R. DeLeo. 2017. Influence of microbes on neutrophil life and death. Front. Cell. Infect. Microbiol. $7: 159$

Kuhns, D. B., D. A. Long Priel, and J. I. Gallin. 1995. Loss of L-selectin $(\mathrm{CD} 62 \mathrm{~L})$ on human neutrophils following exudation in vivo. Cell. Immunol. 164:306-310.

Linder, M., J. H. Paduch, A. S. Grieger, E. Mansion-de Vries, N. Knorr, C. Zinke, K. Teich, and V. Kromker. 2013. Cure rates of chronic subclinical Staphylococcus aureus mastitis in lactating dairy cows after antibiotic therapy. Berl. Munch. Tierarztl. Wochenschr. 126:291-296.

Lippolis, J. D., T. A. Reinhardt, J. P. Goff, and R. L. Horst. 2006. Neutrophil extracellular trap formation by bovine neutrophils is not inhibited by milk. Vet. Immunol. Immunopathol. 113:248-255.

Long, C., M. R. Hosseinkhani, Y. Wang, P. Sriramarao, and B. Walcheck. 2012. ADAM17 activation in circulating neutrophils follow- 
ing bacterial challenge impairs their recruitment. J. Leukoc. Biol. 92:667-672.

Maddox, J. F., K. M. Aherne, C. C. Reddy, and L. M. Sordillo. 1999. Increased neutrophil adherence and adhesion molecule mRNA expression in endothelial cells during selenium deficiency. J. Leukoc. Biol. 65:658-664.

Mallard, B. A., J. C. Dekkers, M. J. Ireland, K. E. Leslie, S. Sharif, C. L. Vankampen, L. Wagter, and B. N. Wilkie. 1998. Alteration in immune responsiveness during the peripartum period and its ramification on dairy cow and calf health. J. Dairy Sci. 81:585-595.

McDougall, S., S. J. LeBlanc, and A. Heiser. 2017. Effect of prepartum energy balance on neutrophil function following pegbovigrastim treatment in periparturient cows. J. Dairy Sci. 100:7478-7492.

Nagahata, H., H. Kawai, H. Higuchi, K. Kawai, K. Yayou, and C. J Chang. 2011. Altered leukocyte responsiveness in dairy cows with naturally occurring chronic Staphylococcus aureus mastitis. J. Vet. Med. Sci. 73:885-894.

Paape, M. J., D. D. Bannerman, X. Zhao, and J. W. Lee. 2003. The bovine neutrophil: Structure and function in blood and milk. Vet. Res. 34:597-627.

Paape, M. J., and A. J. Guidry. 1977. Effect of fat and casein on intracellular killing of Staphylococcus aureus by milk leukocytes. Proc. Soc. Exp. Biol. Med. 155:588-593.

Paape, M. J., P. M. Rautiainen, E. M. Lilius, C. E. Malstrom, and T. H. Elsasser. 2002. Development of anti-bovine TNF-alpha mAb and ELISA for quantitating TNF-alpha in milk after intramammary injection of endotoxin. J. Dairy Sci. 85:765-773.

Powell, E. J., T. A. Reinhardt, E. Casas, and J. D. Lippolis. 2018. The effect of pegylated granulocyte colony-stimulating factor treatment prior to experimental mastitis in lactating Holsteins. J. Dairy Sci.101:8182-8193.

R Core Team. 2014. R: A language and environment for statistical computing. R Foundation for Statistical Computing, Vienna, Austria. http://www.R-project.org/.

Revelo, X., A. L. Kenny, N. M. Barkley, and M. R. Waldron. 2011. Neutrophils harvested from the blood of dairy cows have impaired reactive oxygen species production and release of extracellular traps during the periparturient period. FASEB J. 25(1 Suppl.):38.38

Rivas, A. L., S. J. Schwager, R. N. Gonzalez, F. W. Quimby, and K. L. Anderson. 2007. Multifactorial relationships between intramammary invasion by Staphylococcus aureus and bovine leukocyte markers. Can. J. Vet. Res. 71:135-144.

Ruiz, R., L. O. Tedeschi, and A. Sepulveda. 2017. Investigation of the effect of pegbovigrastim on some periparturient immune disorders and performance in Mexican dairy herds. J. Dairy Sci. 100:33053317 .

Russell, M. W., and B. Reiter. 1975. Phagocytic deficiency of bovine milk leucocytes: An effect of casein. J. Reticuloendothel. Soc. 18:1-13.

Ryman, V. E., G. M. Pighetti, J. D. Lippolis, J. C. Gandy, C. M. Applegate, and L. M. Sordillo. 2015. Quantification of bovine oxylipids during intramammary Streptococcus uberis infection. Prostaglandins Other Lipid Mediat. 121(Pt. B):207-217.

Shoshani, E., G. Leitner, B. Hanochi, A. Saran, N. Y. Shpigel, and A. Berman. 2000. Mammary infection with Staphylococcus aureus in cows: Progress from inoculation to chronic infection and its detection. J. Dairy Res. 67:155-169.

Sieff, C. A. 1987. Hematopoietic growth factors. J. Clin. Invest. 79:1549-1557.

Silvescu, C. I., and R. Sackstein. 2014. G-CSF induces membrane expression of a myeloperoxidase glycovariant that operates as an E-selectin ligand on human myeloid cells. Proc. Natl. Acad. Sci. USA 111:10696-10701.

Smalley, D. M., and K. Ley. 2005. L-selectin: Mechanisms and physiological significance of ectodomain cleavage. J. Cell. Mol. Med. 9:255-266.

Smits, E., C. Burvenich, A. J. Guidry, R. Heyneman, and A. Massart-Leen. 1999. Diapedesis across mammary epithelium reduces phagocytic and oxidative burst of bovine neutrophils. Vet. Immunol. Immunopathol. 68:169-176.

Takahashi, H., M. Odai, K. Mitani, S. Inumaru, S. Arai, R. Horino, and Y. Yokomizo. 2004. Effect of intramammary injection of rboGM-CSF on milk levels of chemiluminescence activity, somatic cell count, and Staphylococcus aureus count in Holstein cows with S. aureus subclinical mastitis. Can. J. Vet. Res. 68:182-187.

Taponen, S., and S. Pyorala. 2009. Coagulase-negative staphylococci as cause of bovine mastitis - Not so different from Staphylococcus aureus? Vet. Microbiol. 134:29-36.

USDA-APHIS (USDA Animal and Plant Health Inspection Service). 2007. Highlights of Dairy2007 Part I: Reference of Dairy Health and Management in the United States. USDA-APHIS, Riverdale, MD.

Van Schyndel, S. J., J. Carrier, O. Bogado Pascottini, and S. J. LeBlanc. 2018. The effect of pegbovigrastim on circulating neutrophil count in dairy cattle: A randomized controlled trial. PLoS One 13:e0198701.

Wang, Y., A. C. Zhang, Z. Ni, A. Herrera, and B. Walcheck. 2010 ADAM17 activity and other mechanisms of soluble L-selectin production during death receptor-induced leukocyte apoptosis. J. Immunol. 184:4447-4454.

Xu, S., M. Hoglund, L. Hakansson, and P. Venge. 2000. Granulocyte colony-stimulating factor (G-CSF) induces the production of cytokines in vivo. Br. J. Haematol. 108:848-853.

Xyni, K., D. Rizos, G. Giannaki, A. Sarandakou, I. Phocas, and G. Creatsas. 2000. Soluble form of ICAM-1, VCAM-1, E- and L-selectin in human milk. Mediators Inflamm. 9:133-140.

Yeiser, E. E., K. E. Leslie, M. L. McGilliard, and C. S. Petersson-Wolfe. 2012. The effects of experimentally induced Escherichia coli mastitis and flunixin meglumine administration on activity measures, feed intake, and milk parameters. J. Dairy Sci. 95:4939-4949.

Zinicola, M., H. Korzec, A. G. V. Teixeira, E. K. Ganda, L. Bringhenti, A. Tomazi, R. O. Gilbert, and R. C. Bicalho. 2018. Effects of pegbovigrastim administration on periparturient diseases, milk production, and reproductive performance of Holstein cows. J. Dairy Sci. 101:11199-11217.

Zöllner, O., M. C. Lenter, J. E. Blanks, E. Borges, M. Steegmaier, H. G. Zerwes, and D. Vestweber. 1997. L-selectin from human, but not from mouse neutrophils binds directly to E-selectin. J. Cell Biol. 136:707-716. 